The authors used the Swedish Cancer register to identify 293 men with testicular germ cell cancer. The pregnancy, delivery and neonatal records for these individuals, as well as for 861 controls, were obtained from the Swedish medical birth register.

The risk of testicular cancer was almost twothirds lower in men who had been exposed to severe maternal hypertension (adjusted odds ratio [OR] $0.29,95 \% \mathrm{Cl} 0.12-0.74$ ) or severe preeclampsia (adjusted OR $0.27,95 \% \mathrm{Cl}$ 0.06-1.17) compared with men who had not been exposed to either of these gestational complications. The risk of testicular cancer was increased, however, among the sons of women who experienced mild gestational hypertension (adjusted OR 1.62, 95\% Cl 0.982.69) or mild preeclampsia (adjusted OR 1.31, 95\% Cl 0.56-3.03). Neither gestational duration nor birth weight had any effect on the risk of testicular cancer.

These results indicate that the low fetal exposure to pregnancy hormones in mothers with severe hypertension and preeclampsia might have a protective effect against testicular cancer.

Original article Pettersson A et al. (2008) Gestational hypertension, preeclampsia, and risk of testicular cancer. Cancer Res 68: 8832-8836

\section{In vivo growth of a mouse prostate gland from a single stem cell}

A US study has demonstrated that a mouse prostate gland can be grown in vivo from a single implanted adult stem cell. A potential population of human prostate stem cells was also identified in the study, which raises the possibility of eventually replacing diseased human prostate glands by stem cell therapy.

The region of the gland proximal to the urethra has been postulated to be the location of prostate stem cells in mice; in this study, Leong et al. established that a number of known stem cell markers were preferentially expressed in the proximal region of the prostate, and also identified a novel potential stem cell marker, CD117. The researchers established that prostate stem cells were contained within the $\mathrm{Lin}^{-}$ Sca $1^{+} \mathrm{CD} 133^{+} \mathrm{CD} 44^{+} \mathrm{CD} 117^{+}$population, which comprises $0.12 \%$ of the viable cell population of the mouse prostate. Engraftment of single donor Lin ${ }^{-S} \mathrm{Sca} 1{ }^{+} \mathrm{CD} 133^{+} \mathrm{CD} 44^{+} \mathrm{CD} 117^{+}$cells in combination with rat urogenital sinus mesenchymal stromal cells under the renal capsule of athymic nu/nu mice generated 14 functioning prostate glands from 97 single-cell transplants. Genotyping confirmed that the generated prostates originated from the donor stem cells.

Leong and colleagues also looked for $\mathrm{CD} 117^{+}$ cells in human prostate samples; they identified a population of $\mathrm{CD} 133^{+} \mathrm{CD} 44^{+} \mathrm{CD} 117^{+}$ cells at a frequency of $0.004 \%$ and $0.01 \%$ in nonbenign prostatic hyperplasia and benign prostatic hyperplasia samples, respectively. Such cells might represent a population of human prostate stem cells.

Original article Leong KG et al. (2008) Generation of a prostate from a single adult stem cell. Nature [doi:10.1038/ nature07427]

\section{Laparoscopic nephrectomy can cause testicular pain}

Testicular pain has been reported as a potential adverse effect of laparoscopic renal surgery, but is generally only identified after direct questioning. Gjertson and Sundaram, therefore, prospectively studied male patients scheduled for laparoscopic kidney and adrenal surgery to determine the incidence of testicular pain postsurgery.

The researchers assessed 64 men (mean age 50 years) in which a total of 68 procedures (60 unilateral, 4 bilateral) were carried out. Initial postoperative physical examination revealed no changes from baseline, and no patient described testicular pain. After discharge from the hospital, 14 patients developed testicular pain (4-7 days after surgery), which was ipsilateral to the surgical side in 13 cases. All patients reporting pain underwent either donor nephrectomy or radical nephrectomy. These men were a mean 10 years younger, had an increased intraoperative urine output, and were less likely to have a history of surgery, than those who experienced no pain. However, the authors were unsure whether these factors were linked to testicular pain or reflected the limited cohort size. Instead, they explained that ligation of the gonadal vein seemed to be associated with the risk of pain: testicular pain developed after $13(33 \%)$ of the 39 procedures where the gonadal vein 